\title{
La discusión controversial, el seminario y aprendizaje significativo en los estudiantes de Maestría de Gestión de la educación y Docencia universitaria 2017-I de la Facultad de Educación UNMSM
}

The controversial discussion, the seminar and significant learning in the students of Master of Management of education and university teaching 2017-I of the Faculty of Education UNMSM

Sonia Quiñones Rodríguez ${ }^{1}$

\section{RESUMEN}

Objetivo: Identificar la relación que existe entre la discusión controversial, el seminario y el aprendizaje significativo en los estudiantes de. Maestría de Gestión de la educación y Docencia universitaria 2017-I de la Facultad de Educación UNMSM. Métodos: La Población de la investigación estuvo conformado por 148 estudiantes ingresantes del 2017-I con una muestra de 40 estudiantes del II ciclo de Maestría Gestión de la Educación y Docencia Universitaria. La selección de la muestra de estudio se efectuó a través de la técnica del muestreo aleatorio simple. El tipo de investigación fue básica descriptiva, explicativa relacional no causales, el diseño de la investigación fue no experimental. Para el análisis de datos se utilizó la estadística Inferencial y para el procesamiento de la información se utilizó el programa estadístico SPSS Versión 24 y la Correlación de Pearson. Resultados: El Coeficiente de Correlación de Pearson r= 0,56 aproximadamente representa el $56 \%$ de los datos están relacionados linealmente entre la discusión controversial, el seminario y el aprendizaje significativo en los estudiantes de. Maestría de gestión de la educación y docencia universitaria 2017-I de la Facultad de Educación UNMSM. Conclusiones: La variable $(Y)$ Aprendizaje significativo y la variable $(X 1)$ discusión controversial, estadísticamente están relacionados, es decir el $52 \%$ de los datos tendría relación lineal. La variable (Y)Aprendizaje significativo y la variable (X2) los seminarios estadísticamente están relacionados; es decir en $54,2 \%$ de los datos tendría relación lineal entre las variables de en los estudiantes de Maestría de Gestión de la educación y Docencia universitaria 2017-I de la Facultad de Educación UNMSM. Por tanto, el seminario tiene mayor relación directa con el aprendizaje significativo.

Palabras clave: Estrategias didácticas, discusión controversial, seminario aprendizajes significativos.

\section{ABSTRACT}

Objective: To identify the relationship that exists between the controversial discussion, the seminar and the significant learning in the students of. Master of Management of Education and University Teaching 2017-I of the Faculty of Education UNMSM. Methods: The research population consisted of 148 incoming students from 2017-I with a sample of 40 students from the II cycle of Master's Degree in Educational Management and University Teaching. The selection of the study sample was made through the technique of simple random sampling. The type of research was basic descriptive, explanatory non-causal relational, the design of the research was non-experimental. For the analysis of data, the Inferential statistics was used and for the processing of the information the statistical program SPSS Version 24 and the Pearson Correlation were used. Results: The Pearson Correlation Coefficient $r=0.56$ approximately represents $56 \%$ of the data are linearly related between the controversial discussion, the seminar and the significant learning in the students of. Master of management of education and university teaching 2017-I of the Faculty of Education UNMSM. Conclusions: The variable (Y) Meaningful learning and the variable (X1) controversial discussion are statistically related, that is, $52 \%$ of the data would have a linear relationship. The variable $(Y)$ Significant learning and the variable $(X 2)$ seminar are statistically related; that is to say, in $54.2 \%$ of the data, there would be a linear relationship between the variables of the students of the Master of Management of Education and the University Teaching 2017-I of the Faculty of Education UNMSM. Therefore, the seminar has a greater direct relationship with meaningful learning.

Keywords: Didactic strategies, controversial discussion, seminar significant learning. 


\section{INTRODUCCIÓN}

La educación superior a nivel de post grado tiene como desafío mejorar la calidad educativa, utilizando las herramientas como las estrategias didácticas, específicamente la discusión controversial y el seminario. Sin embargo, pueden existir otros factores para lograr aprendizaje significativo en la enseñanza -aprendizaje. Analizando la investigación, será un aporte fundamental para los docentes de la Universidad Nacional Mayor de San Marcos y otros ya que es necesario que se ponga en práctica en las aulas de nivel superior la discusión controversial, el seminario porque sabemos que existe relación directa en el aprendizaje significativo en el estudiante y con esto no se desmerece la clase magistral de los docentes. El seminario consiste en investigar, buscar información, discutir en colaboración, analizar los hechos, exponer puntos de vista, reflexionar sobre los problemas suscitados y confrontar criterios en un ambiente de ayuda reciproca para poder lograr las conclusiones del tema. Y La discusión controversial como situación de aprendizaje consiste a aprender a debatir y convencer a los demás, cualquiera sea la opinión que se defienda. Esta estrategia permite al estudiante a sustentar y defender sus puntos de vista y así sistematizar su conocimiento y lograr un aprendizaje autónomo.

No obstante en la sociedad contemporánea no basta adquirir nuevos conocimientos de manera significativos, es preciso adquirirlos críticamente. La educación debe tener como objetivo formar una personalidad inquisitiva, flexible, creativa, innovadora, tolerante y liberal. Así mismo cabe señalar a Palmero (2011), el aprendizaje significativo es una visión humanista, que integra los pensamientos, sentimientos y acciones que conducen al engrandecimiento humano.

Por tal motivo, la investigación contribuirá a las grandes necesidades sociales de la comunidad educativa a nivel superior en su conjunto, dado que la educación es un hecho y una actividad eminentemente de carácter social.
Se quiere concientizar y formar a los responsables de la formación de los profesionales fomentando el uso de estrategias didácticas adecuadas y eficientes para el logro de los aprendizajes significativos.

La investigación planteó la siguiente hipótesis: Existe relación directa entre la discusión controversial, el seminario como técnicas y el aprendizaje significativo en los estudiantes de la Maestría de gestión de la educación y evaluación y docencia universitaria 2017-I de la Facultad de Educación UNMSM.

Y finalmente el objetivo de la investigación Identificar la relación que existe entre la discusión controversial, el seminario y el aprendizaje significativo en los estudiantes de. Maestría de gestión de la educación y docencia universitaria 2017-I de la Facultad de Educación UNMSM.

\section{MATERIAL Y MÉTODOS}

El tipo de investigación fue básica descriptiva, explicativa relacional no causal, el diseño de la investigación fue no experimental, ya que indaga la incidencia y los valores en que se manifiestan una o más variables seleccionadas. Para el análisis de datos se utilizó SPSS Versión 24 y la Correlación de Pearson que mide la relación lineal entre dos variables aleatorias cuantitativas.

La población de la presente investigación, estuvo conformada por 148 estudiantes ingresantes del 2017-I de Maestría de la Facultad de Educación, La muestra de tipo aleatorio simple ascendió a 40 estudiantes de la mención de Gestión de la Educación y Docencia Universitaria. Los instrumentos utilizados para la obtención de datos fue la Escala de Likert para las dos variables, La primera variable independiente la discusión controversial, el seminario, estuvo estructurada por 20 ítems con escala de valoración de 1 al 5 , que significa 1totalmente desacuerdo, 2- desacuerdo, 3parcialmente de acuerdo, 4- de acuerdo, 5muy de acuerdo. $Y$ asimismo la segunda 
variable dependiente aprendizaje significativo, estuvo estructurada con 3 dimensiones: experiencia previas, nuevos conocimientos, y relación entre nuevos y antiguos conocimientos, conformada por 12 ítems con una escala de valoración ( 0 a 4 ), que significa 0 -nunca, 1 - pocas veces, 2 medianamente, 3- muchas veces, 4siempre.

\section{RESULTADOS}

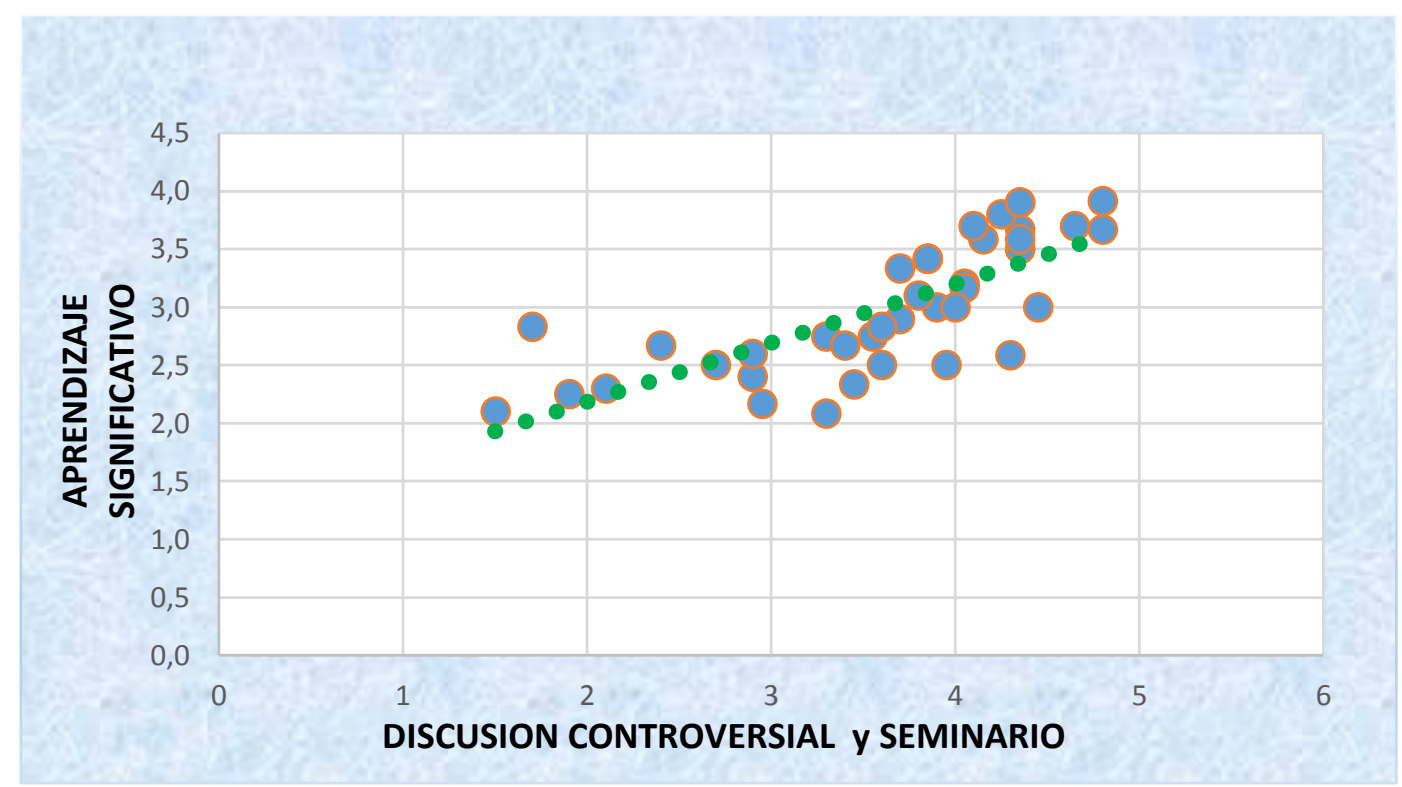

Figura 1. Gráfica de dispersión entre las variables discusión controversial, seminario y variable aprendizaje significativo.

En la Figura 1 se muestra una gráfica de dispersión con una correlación positiva con un cierto grado de sesgo de linealidad entre la discusión controversial, el seminario y el
Respuesta del objetivo general con respecto a la correlación

Identificar la relación que existe entre la discusión controversial, el seminario y el aprendizaje significativo en los estudiantes de. Maestría de Gestión de la educación y Docencia universitaria 2017-I de la Facultad de Educación UNMSM

Tabla 1. Cuadro Estadístico de Correlación entre las variables discusión controversial, seminario y variable aprendizaje significativo

\begin{tabular}{lc}
\hline \multicolumn{2}{c}{ Cuadro Estadístico de Correlación } \\
\hline Coeficiente de correlación múltiple & $\mathbf{0 , 5 5 9}$ \\
Coeficiente de determinación $\mathrm{R}^{\wedge} 2$ & $\mathbf{0 , 3 1 3}$ \\
$\mathrm{R}^{\wedge} 2$ ajustado & 0,294 \\
Error típico & 0,468 \\
Observaciones & 40 \\
\hline
\end{tabular}


El Coeficiente de Correlación de Pearson $r=0,56$ aproximadamente representa el $56 \%$ de los datos están relacionados linealmente entre la discusión controversial, el seminario y el aprendizaje significativo en los estudiantes de Maestría de Gestión de la educación y Docencia universitaria 2017I de la Facultad de Educación UNMSM.
Respuesta al objetivo específico 01

Identificar la relación que existe entre la discusión controversial y el aprendizaje significativo en los estudiantes de maestría de Gestión de la educación y Docencia universitaria 2017-I de la Facultad de Educación UNMSM.

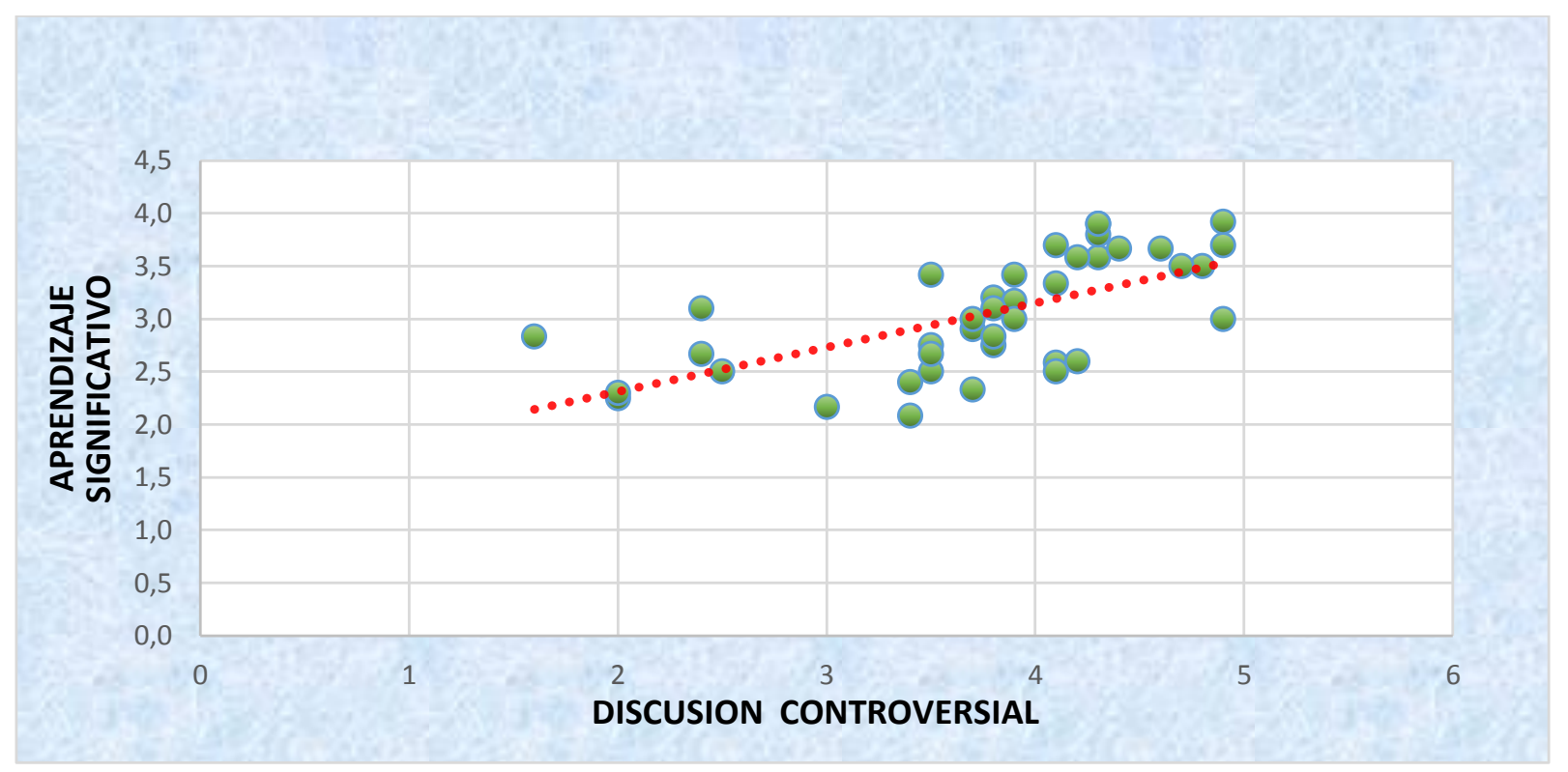

Figura 2. Gráfica de dispersión entre las variables discusión controversial y variable aprendizaje significativo

En la Figura 2 se muestra una gráfica de dispersión con una correlación positiva con un cierto grado de sesgo de linealidad entre las variables la discusión controversial y el aprendizaje significativo en los estudiantes de Maestría de Gestión de la educación y Docencia universitaria 2017-I de la Facultad de Educación UNMSM.

Tabla 2. Cuadro Estadístico de Correlación entre las variables discusión controversial y variable aprendizaje significativo

\begin{tabular}{lc}
\hline \multicolumn{2}{c}{ Estadístico de Correlación } \\
\hline Coeficiente de correlación múltiple & 0,5153 \\
Coeficiente de determinación $\mathrm{R}^{\wedge} 2$ & 0,27 \\
$\mathrm{R}^{\wedge} 2$ ajustado & 0,2463 \\
Error típico & 0,4838 \\
Observaciones (muestra ) & 40 \\
\hline
\end{tabular}

El Coeficiente de Correlación de Pearson $r=$ 0,52 aproximadamente, esto representa el $52 \%$ de los datos están relacionados linealmente entre la variable de la discusión controversial y el aprendizaje significativo en los estudiantes de Maestría de Gestión de la educación y Docencia universitario 
2017-I de la Facultad de Educación UNMSM.

Respuesta al objetivo específico 02
Identificar la relación que existe entre el seminario y el aprendizaje significativo en los estudiantes de maestría de Gestión de la educación y Docencia universitaria 2017I de la Facultad de Educación UNMSM.

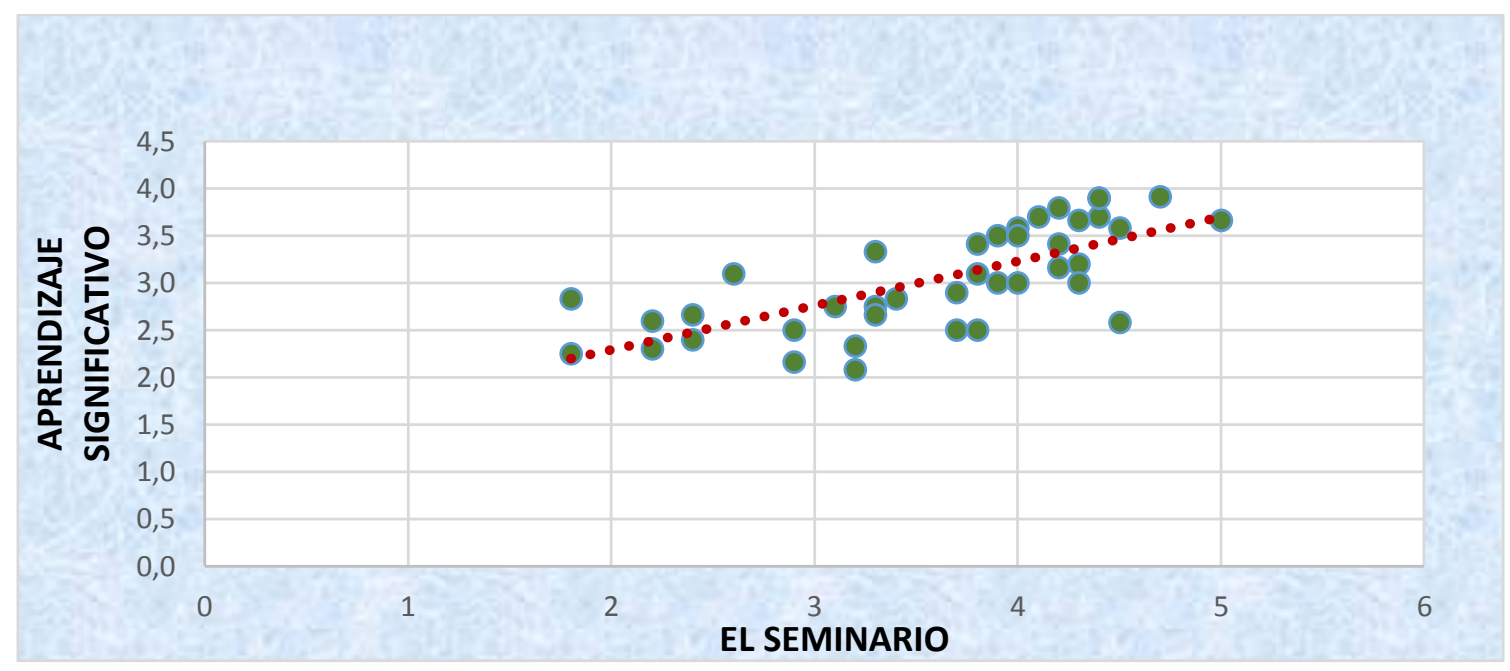

Figura 3. Gráfica de dispersión entre las variables el seminario y aprendizaje significativo

En la Figura 3 se muestra una gráfica de dispersión con una correlación positiva con un cierto grado de sesgo de linealidad entre las variables el seminario y el aprendizaje significativo en los estudiantes de Maestría de gestión de la educación y docencia Universitaria 2017-I de la Facultad de Educación UNMSM.

Tabla 3. Cuadro Estadístico de Correlación entre la variable el seminario y el aprendizaje significativo

\begin{tabular}{ll}
\hline \multicolumn{2}{c}{ Estadístico de Correlación } \\
\hline Coeficiente de correlación múltiple & $\mathbf{0 , 5 4 2 0}$ \\
Coeficiente de determinación $\mathbf{R}^{\wedge 2}$ & $\mathbf{0 , 2 9 3 8}$ \\
$\mathrm{R}^{\wedge} 2$ ajustado & 0,2752 \\
Error típico & 0,4745 \\
Observaciones ( muestra ) & 40 \\
\hline
\end{tabular}

El Coeficiente de Correlación de Pearson $\mathrm{r}=$ 0,5420 aproximadamente representa I $54,2 \%$ de los datos están relacionados linealmente entre la variable el seminario y el aprendizaje significativo en los Estudiantes de Maestría de gestión de la educación y docencia Universitaria 2017-I de la Facultad de Educación UNMSM.

\section{DISCUSION}

En relación a la hipótesis general, los resultados el Coeficiente de Correlación de Pearson $r=0,559$ aproximadamente representa el $56 \%$ de los datos están relacionados linealmente entre la variable discusión controversial, el seminario y el 
aprendizaje significativo en los estudiantes de Maestría de Gestión de la educación y Docencia universitaria 2017-I de la Facultad de Educación UNMSM. Las diferentes incidencias que producen tanto la discusión controversial y el seminario como estrategias didácticas contribuyen favorablemente en el logro de aprendizajes significativos en los estudiantes de la Maestría II ciclo de la Facultad de Educación de la Universidad Nacional Mayor de San Marcos. Al respecto

uso, los docentes están capacitados para su aplicación.

Por otro lado con respecto a la primera hipótesis específica, se establece que el Coeficiente de Correlación de Pearson $r=$ 0,5153 aproximadamente representa el $52 \%$ de los datos están relacionados linealmente entre la discusión controversial y el aprendizaje significativo en los estudiantes de Maestría de Gestión de la educación y Docencia universitaria 2017-I de la Facultad de Educación UNMSM $(\mathrm{H} 1)$ que señala: Existe relación directa entre la discusión controversial y el aprendizaje significativo en los estudiantes de Maestría de Gestión de la educación y Docencia universitaria 2017-I de la Facultad de Educación UNMSM

Corroborando la investigación Quispe (2011) en su tesis titulada "el método de la discusión controversial en el aprendizaje de la asignatura de lenguaje en los estudiantes del II semestre de enfermería del Instituto Superior Tecnológico Joaquín Reátegui Medina, Nauta - 2009; se observa el aporte del método de Discusión Controversial muestra su efectividad en el mejoramiento del nivel de aprendizaje de los estudiantes del II Semestre de la carrera de Enfermería del Instituto Superior Tecnológico - Joaquín Reátegui Medina del Distrito de Nauta, demostrándose en la comparación de los promedios alcanzados después de su aplicación con los estudiantes del grupo control.

En relación a la segunda hipótesis específica, El Coeficiente de Correlación de Pearson $r=0,5420$ aproximadamente representa el $54 \%$ de los datos están relacionados linealmente entre el seminario
Domínguez (2011) en su tesis titulada "las estrategias didácticas y su relación con el aprendizaje de las ciencias sociales en los alumnos de primer año de secundaria de la I.E. Miguel Cortés de Castilla, 2011"sostienen que las estrategias corresponden a una metodología operativa participativa con lo cual promueven el saber y enseñan a aprender; hacen del educando un sujeto disciplinado, creativo y original. Aunque con ciertas limitaciones de orden económico para su elaboración y

y el aprendizaje significativo en los estudiantes de Maestría de Gestión de la educación y Docencia universitaria 2017-I de la Facultad de Educación UNMSM

Por lo que se acepta la hipótesis alterna que señala: Existe relación directa entre el seminario como técnica y el aprendizaje significativo en los estudiantes de Gestión de la educación y Docencia universitaria 2017-I de la Facultad de Educación UNMSM. Como en el caso de Sanabria (2003), en su tesis titulada Influencia del seminario y la clase magistral en el rendimiento académico de alumnos de la E.A.P de Economía de UNMSM Manifiesta que existe la evidencia empírica que el índice académico que se obtiene aplicando el método didáctico de Seminario difiere significativamente al índice académico obtenido con la aplicación del método didáctico de Clase Magistral. A si mismo Gutiérrez (2011) en su tesis titulada la comprensión lectora inferencial y el aprendizaje significativo de los estudiantes de la facultad de ciencias de la Educación y Humanidades de la Universidad "San Luis Gonzaga de Ica" el aprendizaje significativo es una posibilidad que aún no se ha materializado como modelo en la formación profesional. Sin embargo los estudiantes se encuentran en las categorías muy bueno y bueno nivel de comprensión.

Se concluye que el Coeficiente de Correlación de Pearson $r=0,56$ aproximadamente representa el $56 \%$ de los datos están relacionados linealmente entre la discusión controversial, el seminario y el aprendizaje significativo en los estudiantes de. Maestría de gestión de la educación y docencia universitaria 2017-I de la Facultad de Educación UNMSM. 


\section{AGRADECIMIENTO}

A los estudiantes de Maestría de Gestión de la Educación y Docencia Universitaria del II ciclo de la Facultad de Educación, Universidad Nacional Mayor de San Marcos, a sus docentes y autoridades, por haber contribuido desinteresadamente en la realización de la presente investigación.

\section{REFERENCIAS BIBLIOGRÁFICAS}

Domínguez, Z. (2011). Las estrategias didácticas y su relación con el aprendizaje de las ciencias sociales en los alumnos de primer año de secundaria de la IE Miguel Cortés de Castilla. Instituto De Investigación Y Promoción Para El Desarrollo, Universidad Nacional del Piura, Perú.

Gutiérrez Romero, C. P. (2011). La Comprensión lectora inferencial y el aprendizaje significativo de los estudiantes de la Facultad de Ciencias de la Educación y Humanidades de la Universidad Nacional "San Luis Gonzaga" de Ica. Tesis para optar el grado de Magíster en Educación. Universidad Nacional Mayor de San Marcos. Lima, Perú.

Palmero, M. L. R. (2011). La teoría del aprendizaje significativo: una
Quispe, J. (2011). El método de la discusión controversial en el aprendizaje de la asignatura de Lenguaje en los estudiantes del II Semestre de Enfermería del Instituto Superior Tecnológico Joaquín Retegui Medina, Nauta-2009. Tesis para optar el grado de Magíster en Educación. Universidad Nacional Mayor de San Marcos. Lima, Perú.

Sanabria, M. (2003). Influencia del Seminario y La Clase Magistral en el Rendimiento Académico de Alumnos de la EAP de Economía de la UNMSM. Tesis para optar el grado de Magíster en Educación. Universidad Nacional Mayor de San Marcos. Lima, Perú.

revisión aplicable a la escuela actual. IN. Investigació $i$ Innovació Educativa i Socioeducativa, 3(1), 2950.

\section{Correo electrónico:}

almendra.consultora@gmail.com

Revisión de pares:

Recibido:

Aceptado: 